\title{
Построение сбалансированной системы показателей как эффективного средства финансового механизма управления на предприятиях автомобильной промышленности
}

\author{
Курилова А.А. ${ }^{10}$
}

\begin{abstract}
Вопрос эффрективного управления предприятием является актуальным для большинства организаџий. Необходимость принятия во внимание как стратегических, так и тактических иелей деятельности делает вопрос учета всех существенных аспектов деятельности организации актуальным. В статье рассматривается сбалансированная система показателей и практическое внедрение такой системы на примере дочернего предприятия $O A O$ «АвтоВАЗ».
\end{abstract}

\section{JEL: G30, M21}

Ключевые слова: сбалансированная система показателей, SWOT-анализ, финансы, автомобильная промышленность, стратегические иели, индикаторы

$\mathrm{XX}$ век прошел под знаком доминирования крупных промышленных корпораций, располагающих огромными материальными активами и имеющих доступ к мировым ресурсам. Такие корпорации, как «Форд», «Дженерал Моторс», «Дюпон», достигали успеха за счет того, что воплощали новые технологии в физические активы, которые обеспечивали эффективное производство стандартизированных продуктов. В качестве основного измерителя финансовой эффективности компании и создания ценности для собственников использовался показатель прибыли к рабочему капиталу (ROCE).

Информационная эра вносит кардинальные коррективы в создание долгосрочных конкурентных преимуществ, заставляет по-новому взглянуть на стоимость бизнеса и структуру капитала компании. Если в начале 1980-х годов рыночная стоимость западных компаний на 60 и более процентов состояла из материальных активов, то к концу 2000 года их доля снизилась до 10-15\% (по данным компании «Про-Инвест Консалтинг»).

Нематериальные активы (торговые марки, патенты, ноу-хау, качество персонала, деловая репутация, способность к инновациям) обеспечивают компании возможность управлять отношениями с клиентами, создавать новые продукты и услуги непосредственно под запросы целевого сегмента. Более того, они в значительной степени помогают формировать эти запросы, производить качественный продукт при низкой себестоимости и коротком жизненном цикле, использовать навыки и мотивацию персонала для постоянного улучшения внутренних и внешних компетенций организации (Фридаг, Шмидт, 2007).

Принципиальные изменения условий функционирования бизнеса диктуют необходимость формирования новых подходов к постановке системы стратегического управления в компании. Прошлый опыт, интуиция - все, на чем раньше базировались стратегические решения (при всей их ценности), - перестают эффективно работать в постиндустриальном обществе.

Равно как анализ бухгалтерского баланса не дает возможности точно оценить рыночную стоимость нематериальных активов (сколько стоит доверие клиентов?), так и классические инструменты стратегического анализа важны лишь в качестве первичной, грубой настройки. Они не покажут инновационный потенциал компании, ее способности эффективно реагировать на изменения внешней среды. Тем более они не дают точной привязки стратегических решений к финансовым результатам, проекции стратегических

\footnotetext{
10 Канд. эконом. наук, доцент кафедры «Экономика, финансы и кредит» ГОУ ВПО «Тольяттинский государственный университет».
} 
задач на уровень оперативного управления.

Количество неудач и провалов в осуществлении стратегических планов компаний не уменьшилось, несмотря на то что стратегии становятся все сложнее и учитывают все больше факторов, влияющих на состояние дел компании. Почему это происходит? Почему компании не достигают поставленных целей?

В 1999 году журнал Fortune, желая выяснить причины неудач топ-менеджеров, опубликовал результаты собственного исследования, проведенного среди руководителей компаний. Оказалось, что только $10 \%$ компаний успешно реализуют собственную стратегию и достигают поставленных целей. А причиной большинства неудач является не низкое качество самих стратегий, а многочисленные ошибки в их реализации - в первую очередь несогласованность стратегических планов с ежедневной деятельностью сотрудников компаний.

Согласно данным исследования Harvard Business School, среди 937 мировых компаний из перечня Global-1000 основными причинами провалов в реализации стратегии стали:

1. Неправильная реализация стратегических целей компании. В $97 \%$ случаев проваленных или стратегических планов и целей причина - их неправильная реализация.

2. Персонал не знает или не связывает стратегические цели компании со своей деятельностью. 93\% персонала не связывают результаты своей работы со стратегическими целями компании.

3. Распределение ресурсов в планах и бюджетов не имеет связи с реализацией стратегических целей компании. Основной бюджет $73 \%$ компаний связан с обеспечением повседневной деятельности, а не целями предприятия.

4. Контроль деятельности охватывает далеко не все важные для ведения бизнеса показатели. Только $15 \%$ показателей эффективности, используемых компаниями, связаны с необходимостью достижения стратегических целей.

5. Мотивация сотрудников направлена на обеспечение текущих финансовых показателей. Всего 24\% менеджеров мотивированы в своей деятельности на достижение стратегических целей компании.

6. Программы развития бизнеса не учитывают фактора реализации стратегии компании. Только 18\% внутрикорпоративных программ развития направлены на выполнение стратегии развития компании.

Сегодня гораздо труднее добиться конкурентного преимущества за счет эффективного финансового менеджмента и инвестиций в физические активы. Способность компании мобилизовать и использовать свои нематериальные активы стала более значимым фактором. Стратегия фирмы становится как никогда важной. Именно поэтому построение организации, ориентированной на покупателя, и построение бизнеса, ориентированного на стратегию, являются одними из основных инициатив, предпринимаемых большинством динамичных и развивающихся компаний по всему миру. В информационный век в условиях жесточайшей рыночной конкуренции намного большее значение имеет долгосрочное развитие, основными факторами которого являются грамотное стратегическое управление, эффективность бизнеспроцессов, капитал компании, воплощенный в знаниях и квалификации сотрудников, способность организации удерживать и привлекать новых клиентов, корпоративная культура, поощряющая инновации и организационные улучшения, инвестиции в информационные технологии (Каплан, Нортон, 2003).

Системы оценки ориентированы на контроль краткосрочной операционной эффективности, а не долгосрочной стратегии. В среднем $45 \%$ управляющих не тратят ни минуты времени на обсуждение и принятие стратегических решений, 85\% команд управленцев тратят на это менее одного часа в месяц.

Как показали результаты исследования, около 50\% компаний строят системы оценки эффективности, базируясь исключительно на финансовых показателях. Ярким доказательством ущербности такого подхода служит корпорация Xerox, которая, являясь 
одной из крупнейших мировых корпораций по производству копировальной техники, в конце 1970-х годов понесла ощутимые убытки, едва не приведшие к разорению этого гиганта. Первоначально Xerox сдавала в аренду копировальные аппараты, зарабатывая на каждой копии, сделанной с их помощью. Высокие затраты на бумагу, заправку картриджей, ремонт машин, а также выбранная стратегия привели к тому, что клиенты не понимали, почему они должны нести ощутимые издержки из-за оборудования, которое плохо работает и постоянно ломается. Вместо того чтобы изменить стратегию, управляющие Хеrox сосредоточились на улучшении финансовых показателей, не обращая внимания на растущее недовольство клиентов. Руководство компании приняло решение о переходе на новую стратегию, связанную с продажей техники и организацией множества сервисных центров, что, естественно, привело к существенному увеличению затрат. Данная стратегия содействовала достижению корпоративных целей, связанных с ростом прибыли и повышением рентабельности капитала, но только в краткосрочной перспективе. Вследствие того что сервисные центры не успевали обслуживать клиентов, многим из них приходилось покупать несколько машин, тем самым приумножая финансовые результаты Хerox. Естественно, такая ситуация не могла удовлетворить большинство клиентов компании - они хотели иметь относительно недорогие аппараты, более надежные в эксплуатации и производящие более качественные копии. С приходом на рынок американских и японских конкурентов Xerox начала стремительно терять свои позиции. Новички уделяли большое внимание качеству копий, их машины были более надежными и менее дорогостоящими. Таким образом, Хеrox полностью потеряла былое преимущество и была вытеснена с рынка. Только в начале 1980-х годов, с приходом нового руководства, поставившего во главу угла корпоративной стратегии улучшение качества продукции и повышение уровня удовлетворения потребностей клиентов, компании постепенно удалось частично восстановить свои позиции.

Balanced Scorecard (BSC), или Сбалансированная система показателей (CСП), - система управления, позволяющая руководителям переводить стратегические цели компании в четкий план оперативной деятельности подразделений и ключевых сотрудников и оценивать результаты их деятельности с точки зрения реализации стратегии с помощью ключевых показателей эффективности.

Сбалансированная система показателей была создана в 1992 году профессорами Гарвардской школы экономики Дэвидом Нортоном (David Norton) и Робертом Капланом (Robert Kaplan) на основе исследований американских компаний. «Balanced» указывает на «сбалансированность». «Scorecard» дословно - это карточка игрока в гольф. Другими словами, небольшой листок бумаги, на котором в сжатом, агрегированном виде представлена самая важная для игрока информация. Американизм «Balanced Scorecard» подвергся многочисленным попыткам перевода. В разнообразных источниках информации (книгах, журналах, Интернете) можно встретить до десятка вариантов перевода этого термина на русский язык: «сбалансированная система оценочных индикаторов», «сбалансированная счетная карта», «система взаимосвязанных показателей», «сбалансированная система показателей эффективности» и т.д. (Шмидт, Хервиг, Фридаг, 2010).

BSC была создана, когда ряд консалтинговых фирм в США приступил к изучению вопроса эффективности управления, поиску возможностей и инструментов, обеспечивающих ее повышение, а также новых альтернативных методов измерения эффективности, базирующихся на нефинансовых показателях. BSC основывается на комплексном подходе к оценке как материальных, так и нематериальных активов, поэтому, на наш взгляд, название системы следует переводить как сбалансированная система показателей.

Balanced Scorecard делает акцент на нефинансовых показателях эффективности, давая возможность оценить такие, казалось бы, с трудом поддающиеся измерению аспекты деятельности, как степень лояльности клиентов или инновационный потенциал компании.

BSC используется как основной инструмент управления бизнесом, дающий 
возможность устанавливать индивидуальные и общекорпоративные цели, доводить их до сведения сотрудников и управленцев различного уровня, оценивать достижимость поставленных целей посредством использования сбалансированной системы измерителей эффективности, получать быструю обратную связь. Balanced Scorecard стала чрезвычайно удобной моделью, дающей новые аналитические возможности более традиционной концепции MOS (Mission, Objectives, Strategies).

Авторы Balanced Scorecard предложили четыре блока оценки эффективности, отвечающие на самые значимые для успешной деятельности компании вопросы (Каплан, Нортон, 2010):

- Какой компания представляется своим акционерам и потенциальным инвесторам? (Проекция финансов.)

- Какой компания представляется своим покупателям? (Проекция маркетинга.)

- Какие бизнес-процессы компания должна улучшить, от каких отказаться, на каких сосредоточиться? (Проекция внутренних бизнес-процессов.)

- Может ли компания продолжать свое развитие, повышать эффективность и увеличивать свою стоимость? (Проекция обучения и роста.)

В рамках сбалансированной системы необходимо различать показатели, которые измеряют достигнутые результаты, и показатели, которые отражают процессы, способствующие получению этих результатов. Обе категории показателей должны быть увязаны друг с другом, так как для достижения первых (например, определенного уровня производительности) нужно реализовать вторые (например, добиться известной загрузки мощностей машин и оборудования). На практике внимание менеджеров обычно фокусируется на показателях первой категории.

Сбалансированная система показателей охватывает стратегически важные темы. Текущие, так называемые диагностические, сопоставления фактических и намеченных показателей являются предметом других информационных систем. Но на практике границы здесь размыты.

Balanced Scorecard позволяет:

- Оценить стратегию и цели.

- Устранить разрыв между разработкой стратеги и ее воплощением. Благодаря системе взаимосвязанных показателей стратегия предприятия воплощается в жизнь. Одновременно можно оценить обратное воздействие, а именно, понять, как новый проект способен повлиять на достижение стратегических целей.

- Оперативно реагировать на изменения окружающей среды. Все изменения в окружающей среде могут быть оценены с точки зрения их влияния на достижение стратегических целей. При этом может быть использован какой-либо количественный измеритель, например, в рамках инновационного направления.

- Оценить успешность проекта на стадии его возникновения. Когда уже установлены взаимосвязи между различными параметрами развития не трудно понять, есть ли смысл в реализации данного проекта, и насколько это приблизит компанию к достижению стратегических целей.

- Оценить стратегию. Если некое стратегическое решение приводит к большому числу взаимоисключающих воздействий, то, скорее всего, такая стратегия является неудовлетворительной. Кроме того, на основе системы взаимосвязанных показателей возможно подкорректировать существующую стратегию и привести ее в более гармоничное состояние.

- Привязать цели компании к деятельности персонала.

- Увязка оперативного и стратегического менеджмента достигается с помощью многоаспектного и практичного метода.

- Четыре основных аспекта (потребительский, хозяйственный, инновационный и финансовый) образуют всеохватывающую схему для «проводки» сверху вниз стратегии предприятия по всем его иерархическим уровням. 
- Дискуссии по проблеме реализации стратегии приобретают объективный характер в связи с необходимостью отыскания единиц измерения и благодаря так называемой стратегической карте.

- Предлагаемая система управления делает возможной широкую, сориентированную на обучение коммуникацию по всем уровням предприятия.

- Новая концепция удачно интегрируется с системой контроллинга и хорошо увязывается с методами управления, нацеленными на повышение стоимости предприятия.

Обобщая изложенный материал, можно сказать, что BSC дает высшему руководству компании совершенно новый инструмент управления, представляющий собой набор взаимосвязанных сбалансированных показателей, позволяющих оценивать критические факторы не только текущего, но и будущего ее развития.

Используя BSC, менеджеры руководят такими процессами, как перевод видения стратегии, доведение ее до всех уровней системы управления, бизнес-планирование и распределение ресурсов, обратная связь, обучение и текущий мониторинг реализации стратегии.

Кроме того, они получают ответы на основные вопросы: за счет чего компания создает свою стоимость, какие процессы являются ключевыми в ее деятельности, каков уровень квалификации персонала и качество применяемых технологий, каково их соответствие выбранной стратегии, какие инвестиции нужно осуществить в развитие компании с целью достижения ее долгосрочных целей.

B результате использования Balanced Scorecard деятельность любой организации становится более прозрачной и управляемой. Существует еще одна весомая причина, побуждающая компании внедрять BSC. Это растущий интерес к информации, позволяющей оценить будущий потенциал компании со стороны внешних кредиторов и инвесторов.

Рассмотрим примерное (условное) построение системы Balanced Scorecard на одном из дочерних обществ ОАО «АвтоВАЗ».

Этап 1. Разработка базовой стратегической ориентации компании

Компания позиционируется на автомобильном российском рынке, стремясь поставлять продукцию ОАО «АвтоВАЗ», ориентированную на удовлетворение потребностей клиентов.

Предприятие предоставляет полный комплекс услуг, связанных с продажей, гарантийным и сервисным обслуживанием автомобилей для российских потребителей.

Открытая информация на сайте компании описывает миссию, преимущества и принципы деятельности предприятия.

Преимущества:

- Мы работаем на протяжении 30 лет - и это не просто годы, это высокий профессионализм и уникальная система знаний, накопленная за годы работы, традиции качества.

- Мы являемся динамично развивающейся компанией. Стремительно растет число наших филиалов в регионе, увеличивается спектр оказываемых услуг, обновляется технологическая база.

- Являясь авторизованным дилером «АвтоВАЗ» а и специализированным дилерским центром компании GM - AvtoVAZ, мы предоставляем самые долгосрочные гарантии и наиболее низкие цены на автомобили и обслуживание по сравнению с другими автоцентрами в регионе.

- Мы - единственный в регионе специализированный автоцентр, который имеет право проводить гарантийный ремонт автомобилей «Лада» независимо от места их сборки Тольятти, Сызрань или Ижевск.

Наши принципы:

- обеспечение самого высокого уровня обслуживания в России, предоставление полного спектра оригинальных материалов и комплектующих для автомобилей и лучших сервисных услуг в одно время и в одном месте; 
- постоянное повышение профессионального уровня сотрудников компании;

- поддержание в коллективе психологического климата, способствующего проявлению творческой активности и инициативы наших сотрудников.

Базовыми ценностями компании являются непрерывное расширение масштабов деятельности при постоянном повышении качества удовлетворения потребностей клиентов.

Проведенный SWOT-анализ (анализ сильных и слабых сторон, возможностей и угроз) позволил сформулировать основные достижения и проблемы, а также оценить имеющиеся направления развития компании (см. таблицу ниже).

Таблица 1

SWOT-анализ компании

\section{Сильные стороны / Strengths}

- Гибкость оказания услуг

- Предоставление полного услуг, связанных с гарантийным и обслуживанием автомобилей

- Качество оказываемых услуг

- Возможность удовлетворения индивидуальных заказов

- Квалификация кадров, высокий профессионализм

- Самые долгосрочные гарантии и наиболее низкие цены на автомобили и обслуживание по сравнению с другими автоцентрами регионе

- Единственный в регионе специализированных автоцентр, который имеет право проводить гарантийный ремонт автомобилей Лада независимо от места их сборки

\section{Возможности / Opportunities}

- Рост спроса на оказание такого рода услуг

- Рост платежеспособности клиентов на рынке

\section{Угрозы / Theats}

- Активизация конкурентов

- Рост налогов

- Нестабильная экономическая ситуация

Этап 2. Определение стратегических целей, индикаторов и целевых значений индикаторов по перспективе «Финансы / Экономика» (Какие параметры финансового состояния будут приемлемыми для нас и наших учредителей?)

Предложение: «Снизить затраты».

Оценка предложения: Затраты в данном бизнесе - один из решающих факторов. Чтобы выжить на рынке, компания должна прикладывать постоянные усилия к управлению затратами. Проблематичным в отношении формулировки «снизить затраты» является то обстоятельство, что экономичная работа предприятия во все времена и при всех стратегиях является одной из самых важных целей. Другими словами, снижение затрат является само собой разумеющимся фактором успеха. Однако, по нашему мнению, будет правильнее предложить критерий улучшения структуры выручки.

Предложение: «Обеспечить достаточную ликвидность».

Оценка предложения: Достаточная степень ликвидности - необходимое условие успешности оперативной деятельности предприятия. Кроме того, данный индикатор платежеспособности устанавливается заданием к контракту, определяемым материнской компанией ОАО «АвтоВАЗ» . 
Предложение: «Увеличить рентабельность капитала».

Оценка предложения: Фирма может измерять эффективность своего бизнеса такими индикаторами, как рентабельность совокупного капитала - ROI \{(чистая прибыль + проценты)/совокупный капитал\} или рентабельность собственного капитала - ROE \{чистая прибыль/собственный капитал\}. Кроме того, данный индикатор установлен Заданием к контракту, определяемым материнской компанией ОАО «АвтоВАЗ».

Предложение: «Увеличить величину денежного потока (Cash Flow)».

Оценка предложения: Эта цель связана с целью «Обеспечить достаточную ликвидность». Однако коэффициенты ликвидности (1-й, 2-й и 3-й степени) характеризуют платежеспособность предприятия на конкретную дату, но не информируют о движении денежных средств за период. Помимо абсолютного значения денежного потока (Cash Flow) эффективность бизнеса характеризует показатель CFROA (Cash Flow Return on Assets), который рассчитывается как соотношение денежного потока (поступления - выплаты по основной деятельности) и величины активов.

После обсуждения всех формулировок стратегических целей по перспективе «Финансы/Экономика» и расстановки приоритетов для окончательного варианта стратегических целей разрабатываются индикаторы, на основе значений которых руководство будет оценивать степень достижения целей. Каждая стратегическая цель может измеряться одним или несколькими показателями.

Таблица 2

\begin{tabular}{|c|c|}
\hline Стратегическая цель & Индикатор \\
\hline Увеличить рентабельность капитала & ROI, RI, ROE \\
\hline $\begin{array}{l}\text { Добиться конкурентоспособной структуры } \\
\text { выручки }\end{array}$ & $\begin{array}{l}\text { Доля постоянных и переменных затрат в } \\
\text { выручке, показатели рентабельности. } \\
\text { Изменение и сравнение с бенчмакинговой } \\
\text { оценкой }\end{array}$ \\
\hline Обеспечить достаточную ликвидность & Показатели ликвидности \\
\hline $\begin{array}{l}\text { Увеличить величину денежного потока } \\
\text { (CashFlow) }\end{array}$ & Cash Flow Return on Assets \\
\hline
\end{tabular}

Далее индикаторам присваиваются целевые значения (как правило, в динамике). В рассматриваемой компании руководство анализирует полный пакет индикаторов системы Balanced Scorecard раз в полгода (отдельные показатели еженедельно, ежемесячно или ежеквартально). При разработке Balanced Scorecard заполняется следующая таблица:

Таблица 3

\begin{tabular}{|c|c|c|c|}
\hline \multirow[b]{2}{*}{ Стратегическая цель } & \multirow[b]{2}{*}{ Индикатор } & \multicolumn{2}{|c|}{ Целевое значение } \\
\hline & & $\begin{array}{c}1 \\
\text { полугодие }\end{array}$ & $\begin{array}{c}2 \\
\text { полугодие }\end{array}$ \\
\hline 1 & 2 & 3 & 4 \\
\hline$\ldots$ & $\ldots$ & $\ldots$ & $\ldots$ \\
\hline
\end{tabular}

Этап 3. Определение стратегических целей, индикаторов и целевых значений индикаторов по перспективе «Рынок / Клиенты» (как мы должны выглядеть в глазах наших клиентов (по сравнению с конкурентами), чтобы достичь тех финансово-экономических целей, которые мы перед собой поставили?)

Предложение: «Улучшить качество». 
Оценка предложения: Эта цель относится к числу тех общих целей, которые очень часто встречаются в создаваемых Balanced Scorecard. При обсуждении такой цели важно добиться четкой конкретизации - на каких аспектах качества компания должна сконцентрироваться с точки зрения достижения конкурентных преимуществ.

Предложение: «Повысить степень удовлетворенности клиентов».

Оценка предложения: Повышение степени удовлетворенности клиентов предполагает оказание качественных услуг, связанных с продажей, гарантийным и сервисным обслуживанием автомобилей для российских потребителей (в приемлемые сроки и по приемлемым ценам), а также ориентация на индивидуальных покупателей с учетом спектра требуемых материалов и комплектующих для автомобилей и лучших сервисных услуг в одно время и в одном месте.

Предложение: «Увеличить долю рынка».

Оценка предложения: Можно считать, что эта цель уже учтена в перспективе «Финансы / Экономика» (в рамках цели «Увеличить продажи»). Во многих вариантах Balanced Scorecard цели, связанные с выручкой, и цели, связанные с долей рынка, объединяются в некую единую цель (например, «Продолжить рост продаж»). Однако следует учитывать, что рост продаж компании может быть связан как с «отвоеванием» доли рынка у конкурентов, так и с привлечением новых клиентов.

Предложение: «Увеличить число заказов».

Оценка предложения: Разработать стратегию - означает сконцентрироваться на определенных аспектах, а не решать все проблемы сразу. Возможно развитие сбытовой сети и повышении узнаваемости бренда, которые, в свою очередь будут способствовать и привлечению дополнительного объема заказов от клиентов. К тому же индикаторы «Реализация автомобилей» и «Выручка от реализации услуг ТО» устанавливаются Заданием к контракту, определяемым материнской компанией ОАО «АвтоВАЗ».

Таблица 4

\begin{tabular}{|l|l|}
\hline \multicolumn{1}{|c|}{ Стратегическая цель } & \multicolumn{1}{|c|}{ Индикатор } \\
\hline Увеличить число заказов & $\begin{array}{l}\text { Выручка (планируется некоторый рост), Темп роста } \\
\text { совокупной выручки (\%) }\end{array}$ \\
\hline Увеличить долю рынка & $\begin{array}{l}\text { Доля рынка по России и в частности }- \text { по } \\
\text { Поволжью }\end{array}$ \\
\hline Улучшить качество & Количество жалоб и претензий \\
\hline $\begin{array}{l}\text { Повысить степень удовлетворенности } \\
\text { клиента }\end{array}$ & $\begin{array}{l}\text { Индекс удовлетворенности клиентов, объем } \\
\text { повторных продаж }\end{array}$ \\
\hline
\end{tabular}

Этап 4. Определение стратегических целей, индикаторов и целевых значений индикаторов по перспективе «Бизнес-процессы» (Каким критериям (затраты, время, качество) должны соответствовать наши процессы, чтобы мы смогли достичь тех целей, которые мы перед собой поставили по перспективе «Рынок / Клиенты»?).

Предложение: «Ускорить время обработки заказа».

Оценка предложения: Повышению степени удовлетворенности клиентов будет способствовать сокращение времени обработки заказа (согласование технических и финансовых условий, расчет плановой калькуляции, составление договора).

Предложение: «Сохранить гибкость процесса оказания услуг».

Оценка предложения: Рост числа филиалов в Самаре и области, увеличение спектра оказываемых услуг, обновление технологической базы.

Предложение: «Минимизировать входной брак».

Оценка предложения: Предприятие возможно периодически сталкивается с проблемой низкого качества закупаемых комплектующих. 
Предложение: «Снизить стоимость закупок комплектующих».

Оценка предложения: Снижение закупочных цен на ресурс призвано увеличить значение показатели рентабельности реализации (доля прибыли в выручке) и возможно за счет соответствующих переговоров с поставщиками и поиска альтернативных поставщиков. Однако такая деятельность относится к числу «рутинных» задач службы снабжения. Выполнение этой цели необходимо для поддержания нормальной деятельности фирмы. Такого рода базовые требования, как правило, не должны зависеть от используемой фирмой стратегии.

Предложение: «Повысить качество оказываемых услуг».

Оценка предложения: Эта цель уже обсуждалась в рамках перспектив «Финансы / Экономика» и «Рынок / Клиенты». Следует сказать, что качество продукции в настоящее время рассматривается как сильная сторона фирмы (см. SWOT-анализ), однако стремление фирмы к постоянному совершенствованию (continuous improvement) должно быть заложено в базовую стратегическую ориентацию. Кроме того, фирма должна четко осознавать конкретные направления совершенствования качества оказания услуг.

Предложение: «Усовершенствовать систему управленческого учета и бюджетирования».

Оценка предложения: Речь идет, в частности, о повышении степени автоматизации системы управленческого учета и бюджетирования, о привязке системы премирования / депремирования центров затрат к результатам их деятельности (что требует разработки соответствующей системы индикаторов), об изменении политики распределения косвенных затрат по видам производимой продукции.

Таблица 5

\begin{tabular}{|c|c|}
\hline Стратегическая цель & Индикатор \\
\hline Повысить качество оказываемых услуг & $\begin{array}{l}\text { Доля брака, отклонение план/факт по } \\
\text { производственной себестоимости }\end{array}$ \\
\hline $\begin{array}{l}\text { Сохранить гибкость процесса оказания } \\
\text { услуг }\end{array}$ & $\begin{array}{l}\text { Возможность удовлетворения индивидуальных } \\
\text { запросов клиентов с дифференциацией } \\
\text { местности }\end{array}$ \\
\hline $\begin{array}{l}\text { Снизить стоимость } \\
\text { комплектующих }\end{array}$ & Стоимость закупок, доля в общих затратах \\
\hline Ускорить время обработки заказа & Среднее время обработки заказа \\
\hline Минимизировать входной брак & $\begin{array}{lll}\text { Количество жалоб } & \text { и претензий } & \text { на новые } \\
\text { комплектующие, } & \text { количество } & \text { поставок } \\
\text { несоответствующего качества } & \end{array}$ \\
\hline \begin{tabular}{|cr} 
Усовершенствовать & систему \\
управленческого учета и бюджетирования
\end{tabular} & $\begin{array}{l}\text { Возможно применение балльной системы по } \\
\text { специально разработанному перечню критериев } \\
\text { (степень автоматизации, оперативность, } \\
\text { достоверность, возможность получения } \\
\text { информации по нескольким «срезам» } \\
\text { одновременно, наличие информации в разрезе } \\
\text { центров затрат) }\end{array}$ \\
\hline
\end{tabular}

Этап 5. Определение стратегических целей, индикаторов и целевых значений индикаторов по перспективе «Инфраструктура / Инновации / Сотрудники» (Какие инфраструктура и сотрудники нам необходимы для достижения целей по перспективе «Бизнес-процессы»?)

Предложение: «Повысить квалификацию сотрудников». 
Оценка предложения: «Повысить квалификацию» - это одна из самых общих целей, которые предприятия могут использовать в рамках Balanced Scorecard. Повышение квалификации относится к самым общим целям большинства предприятий. Именно поэтому при постановке такой цели следует подумать о том, как именно она вписывается в стратегию. Квалификацию каких именно сотрудников следует повысить? Компания собирается уделить особое внимание, прежде всего, компетенциям своих автомехаников и сотрудников, занимающихся продажами.

Предложение: «Повысить удовлетворенность сотрудников фирмы».

Оценка предложения: Удовлетворенность сотрудников, работающих на фирме.

Предложение: «Реализовать на практике идею непрерывного совершенствования (continuous improvement)».

Оценка предложения: Руководство фирмы считает, что совершенствование компетенций персонала должно быть непрерывным и последовательным. Одно из самых важных требований к сотрудникам, работающим в компании - готовность к изменениям, стремление к постоянному повышению квалификации и социальных компетенций (работа в команде, коммуникабельность, гибкость, креативность). Достижению этой цели будет способствовать, в частности, реализация новых идей (проектов) - внутри как отдельных подразделений, так и межфункциональных (внедрение системы менеджмента качества ISO 9000, повышение степени автоматизации системы управленческого учета и др.).

Предложение: «Сохранить приверженность принципам командной работы и демократизма менеджмента».

Оценка предложения: Система менеджмента фирмы базируется на принципах командной работы (коллегиальное решение проблем, реализация межфункциональных проектов, построение процессно-ориентированной организации, создание кружков качества) и демократизма менеджмента (обсуждение целей с подчиненными, выпуск внутрифирменной газеты, «плоская» организационная структура). Эти принципы рассматриваются как принципиальные (базовые).

Таблица 6

\begin{tabular}{|c|c|}
\hline Стратегическая цель & Индикатор \\
\hline $\begin{array}{l}\text { Повысить } \\
\text { удовлетворенность } \\
\text { сотрудников фирмы }\end{array}$ & $\begin{array}{l}\text { Индекс удовлетворенности сотрудников (на основе } \\
\text { специально разработанного чек-листа, включающего перечень } \\
\text { критериев, важных для удовлетворенности сотрудников, } \\
\text { работающих на предприятии, с присвоением отдельным } \\
\text { критериям весов и расчетом взвешенной сумму баллов по } \\
\text { каждому сотруднику), Число претендентов на ключевые } \\
\text { должности }\end{array}$ \\
\hline $\begin{array}{l}\text { Повысить } \quad \text { квалификацию } \\
\text { сотрудников }\end{array}$ & Затраты на обучение персонала, Рост прибыли \\
\hline $\begin{array}{lr}\text { Сохранить } & \text { приверженность } \\
\text { принципам } & \text { командной } \\
\text { работы и } & \text { демократизма } \\
\text { менеджмента } & \end{array}$ & $\begin{array}{l}\text { Число общих собраний в квартал (коллегиальность работы } \\
\text { измеряется числом собраний (общефирменных, внутри- и } \\
\text { межфункциональных), на которых совместно решаются те или } \\
\text { иные проблемы), индекс удовлетворенности сотрудников }\end{array}$ \\
\hline $\begin{array}{ll}\text { Реализовать } & \text { на практике } \\
\text { идею } & \text { непрерывного } \\
\text { совершенствования }\end{array}$ & $\begin{array}{l}\text { Число рационализаторских предложений, число реализуемых } \\
\text { проектов (фирма стремится к тому, чтобы каждый сотрудник } \\
\text { помимо решения текущих («рутинных») задач был вовлечен в } \\
\text { тот или иной проект) }\end{array}$ \\
\hline
\end{tabular}

Этап 6. Построение причинно-следственной цепочки стратегических целей

После определения стратегических целей по каждой перспективе, разработки 
показателей по каждой цели и присвоения показателям целевых значений выстраивается причинно-следственная цепочка целей компании. На нижнем уровне системы целей располагаются цели перспективы «Инфраструктура / Сотрудники», служащие средством реализации целей перспектив «Бизнес-процессы», «Рынок / Клиенты» и «Финансы / Экономика».

Этап 7. Разработка мероприятий, необходимых для достижения стратегических целей, определение бюджетов, сроков и ответственных за реализацию мероприятий

Разработка системы Balanced Scorecard завершается разработкой мероприятий, реализация которых необходима для достижения поставленных стратегических целей. Для разработанных мероприятий, как правило, определяется бюджет, сроки выполнения и ответственное лицо. Тем самым модель Balanced Scorecard прокладывает «мостик» из стратегического планирования в планирование оперативное. Информацию этапа 7 удобно представлять в виде следующей таблицы:

\begin{tabular}{|l|l|l|l|l||l|l|}
\hline $\begin{array}{c}\text { Cтратегическая } \\
\text { цель }\end{array}$ & Индикаторы & $\begin{array}{c}\text { Целевое } \\
\text { значение }\end{array}$ & Мероприятия & Ответственный & Бюджет & Сроки \\
\hline 1 & 2 & 3 & 4 & 5 & 6 & 7 \\
\hline$\ldots$ & $\ldots$ & $\ldots$ & $\ldots$ & $\ldots$ & $\ldots$ & $\ldots$ \\
\hline
\end{tabular}

Дополнительные возможности использования системы Balanced Scorecard состоят в так называемом «каскадировании» - разбиении системы показателей компании как бизнеса в целом на системы показателей для отдельных подразделений. Если компания построена по дивизиональному принципу или представляет собой холдинговую структуру, то Scorecard'ы разрабатываются для отдельных дивизионов или направлений.

Если компания построена по функциональному принципу, то Scorecard'ы разрабатываются для отдельных структурных подразделений — снабжение, производство, маркетинг / сбыт.

Ниже приводятся несколько критериев отбора, которые, как показал опыт и исследования ведущих консультантов, помогают эффективно оценить и выбрать показатели.

Связь со стратегией. Этот критерий самый явный, но его значение нельзя переоценить. Система показателей - это инструмент перевода стратегии в действия посредством показателей деятельности, рассказывающих о вашей стратегии. Выбор показателей деятельности, не влияющих на реализацию стратегии, может привести к замешательству и путанице, так как работники будут расходовать ценные ресурсы на выполнение показателей, от которых не зависит достижение общих целей фирмы.

Количественное выражение. У специалистов-практиков по системе показателей часто возникает желание включить показатели, подразумевающие субъективную оценку деятельности, например, оценка поставщиков: «хороший», «удовлетворительный» или «средний». Несомненно, основная проблема этого подхода состоит в том, что десять разных людей предложат совершенно разные методы и результаты оценки одного и того же поставщика. Однако если поставщиков оценивают по проценту своевременной доставки продукции, результаты будут объективными и однозначными для всех членов команды.

Доступность. Каплан и Нортон часто обсуждают метрику недостающих показателей. Это такие показатели деятельности, которые вы не зафиксировали в прошлом и которые увидели свет только благодаря процессу разработки сбалансированной системы. Нет сомнения в том, что новые и нетрадиционные показатели являются великолепным преимуществом сбалансированной системы; фактически, недостающие показатели могут указывать на то, что в настоящее время ни один из создающих стоимость процессов не управляется. При этом вы должны избегать включения желаемых показателей деятельности, требующих больших инвестиций в инфраструктуру информационной технологии.

Доходчивость. Читатели сбалансированной системы сразу должны понимать как 
операционное, так и стратегическое значение каждого показателя. Также должно быть очевидным и желаемое направление показателя.

Сбалансированность.

Релевантность. Показатели в вашей системе должны точно отражать процесс или цель, которые вы пытаетесь оценить. Хорошей проверкой является определение возможности действовать на основе результатов данного показателя.

Наличие общего определения. Ваша сбалансированная система, вероятно, будет содержать ряд узкоспециальных показателей деятельности, и это совершенно правильно, так как она рассказывает именно о вашей стратегии. Проблемы возникают тогда, когда вы включаете в систему показатели с расплывчатыми определениями или вообще без определений.

Для успешного внедрения системы Balanced Scorecard экономисты рекомендуют следовать следующим основным правилам:

- Система Balanced Scorecard не должна разрабатываться руководителем в одиночку и втайне от сотрудников компании. Balanced Scorecard - это продукт работы команды.

- Разработка Balanced Scorecard - это достаточно длительный процесс и, скорее всего, вам потребуется определенное количество уточнений и корректировок первого варианта, прежде чем система начнет работать.

- Основная сложность при построении Balanced Scorecard - подбор адекватного показателя (показателей) для измерения той или иной стратегической цели. Та или иная проблема может быть трудноизмеримой вообще (например, готовность персонала к изменениям, атмосфера в коллективе, качество системы управленческого учета, имидж компании). Или же сбор информации для расчета значения того или иного показателя может оказаться чрезмерно дорогим.

Внедрение стратегии начинается с обучения тех, кто должен исполнять ее. С помощью специально разработанных программ, периодических собраний, корпоративных посланий и информационных систем руководство осуществляет функцию коммуникации стратегического видения. Это дает ему возможность проводить стратегию в различных направлениях, обеспечивая тем самым связь общих целей с целями структурных подразделений и функциями отдельных сотрудников.

Коммуникация осуществляется за счет последовательного вовлечения всех отделов и сотрудников в разработку стратегий и сбалансированных показателей, оценивающих ее достижение.

Внедрение BSC на отечественных предприятиях следует осуществлять, используя различные подходы. Применение единого подхода в данном случае невозможно. В качестве критериев выбора того или иного метода внедрения выступают масштабы предприятия, отраслевая специфика, особенности корпоративной культуры, наличие системы бизнеспланирования и стратегического менеджмента, методы управления и инструменты, применяемые руководством, отлаженность бизнес-процессов, эффективность и охват системы управленческого учета. Протяженность проекта по внедрению Balanced Scorecard колеблется от 3 до 24, а иногда и более месяцев. Реорганизация и подготовка предприятия к процессу внедрения может охватить половину срока реализации проекта.

Суммируя, преимущества системы можно выразить следующим образом: сбалансированная система показателей является инструментом, позволяющим полномасштабно увязать стратегию предприятия с оперативным бизнесом; кроме того, система дает возможность принимать вполне объективные решения в области распределения ресурсов.

\section{Список литературы}

1 Каплан Р., Нортон Д. Сбалансированная система показателей. От стратегии к действию. М.: Олимп-Бизнес, 2003. 
2 Каплан Р., Нортон Д. Сбалансированная система показателей. М.: Олимп-Бизнес, 2010.

3 Кини Р.Л., Райфа Х. Принятие решений при многих критериях: предпочтения и замещения: Пер. с англ. М.: Радио и связь, 1981.

4 Консалтинговый портал [Электронный ресурс]: официальный сайт. - Режим доступа: http://www.consult.ru/themes.

5 Шанк Д.К., Говиндараджан В. Стратегическое управление затратами: Пер. с англ. СПб.: ЗАО «Бизнес Микро», 1999.

6 Шмидт В., Хервиг Р. Фридаг Т. Сбалансированная система показателей. М.: Омега-Л, 2010. С. 60-70.

7 Фридаг Х.Р., Шмидт В. Сбалансированная система показателей: руководство по внедрению. М.: Омега-Л, 2007. С. 125-127.

8 Технологии корпоративного управления [Электронный ресурс]: официальный сайт. Режим доступа: http://www.iteam.ru. 